\title{
Estimating the incidence and diagnosed proportion of HIV infections in Japan: a statistical modeling study
}

\author{
Hiroshi Nishiura Corresp. 1,2 \\ ${ }^{1}$ Graduate School of Medicine, Hokkaido University, Sapporo, Japan \\ 2 CREST, Japan Science and Technology Agency, Saitama, Japan \\ Corresponding Author: Hiroshi Nishiura \\ Email address: nishiurah@gmail.com
}

Background. Epidemiological surveillance of HIV infection in Japan involves two technical problems for directly applying a classical backcalculation method, i.e., (i) all AIDS cases are not counted over time and (ii) people diagnosed with HIV have received antiretroviral therapy, extending the incubation period. The present study aimed to address these issues and estimate the HIV incidence and the proportion of diagnosed HIV infections, using a simple statistical model.

Methods. From among Japanese nationals, yearly incidence data of HIV diagnoses and patients with AIDS who had not previously been diagnosed as HIV positive, from 1985 to 2017, were analyzed. Using the McKendrick partial differential equation, general convolution-like equations were derived, allowing estimation of the HIV incidence and the time-dependent rate of diagnosis. A likelihood-based approach was used to obtain parameter estimates.

Results. Assuming that the median incubation period was 10.0 years, the cumulative number of HIV infections was estimated to be 29,613 (95\% confidence interval (CI): $29,059,30,167$ ) by the end of 2017 , and the proportion of diagnosed HIV infections was estimated at $80.3 \%$ (95\% Cl: $78.7 \%, 82.0 \%)$. Allowing the median incubation period to range from 7.5 to 12.3 years, the estimate of the proportion diagnosed can vary from $77 \%$ to $84 \%$.

Discussion. The proportion of diagnosed HIV infections appears to have not yet reached $90 \%$ among Japanese nationals. Compared with the peak incidence from 2005-2008, new HIV infections have clearly been in a declining trend; however, there are still more than 1,000 new HIV infections per year in Japan. To increase the diagnosed proportion of HIV infections, it is critical to identify people who have difficulty accessing consultation, testing, and care, and to explore heterogeneous patterns of infection. 
1 Estimating the incidence and diagnosed proportion of HIV infections in Japan: a statistical

2 modeling study

3

4 Hiroshi Nishiura ${ }^{1,2}$

5

$6{ }^{1}$ Graduate School of Medicine, Hokkaido University, Sapporo, Japan

$7 \quad{ }^{2}$ CREST, Japan Science and Technology Agency, Saitama, Japan

8

9 Corresponding Author:

10 Hiroshi Nishiura

11 Graduate School of Medicine, Hokkaido University, Sapporo, Japan

12 Kita 15 Jo Nishi 7 Chome, Kita-ku, Sapporo-shi, Hokkaido 060-8638, Japan

13 Email address: nishiurah@med.hokudai.ac.jp 


\section{Abstract}

16 Background. Epidemiological surveillance of HIV infection in Japan involves two technical

17 problems for directly applying a classical backcalculation method, i.e., (i) all AIDS cases are not

18 counted over time and (ii) people diagnosed with HIV have received antiretroviral therapy,

19 extending the incubation period. The present study aimed to address these issues and estimate the

20 HIV incidence and the proportion of diagnosed HIV infections, using a simple statistical model.

21 Methods. From among Japanese nationals, yearly incidence data of HIV diagnoses and patients

22 with AIDS who had not previously been diagnosed as HIV positive, from 1985 to 2017, were

23 analyzed. Using the McKendrick partial differential equation, general convolution-like equations

24 were derived, allowing estimation of the HIV incidence and the time-dependent rate of

25 diagnosis. A likelihood-based approach was used to obtain parameter estimates.

26 Results. Assuming that the median incubation period was 10.0 years, the cumulative number of

27 HIV infections was estimated to be 29,613 (95\% confidence interval (CI): $29,059,30,167)$ by the

28 end of 2017, and the proportion of diagnosed HIV infections was estimated at $80.3 \%$ (95\% CI:

$2978.7 \%, 82.0 \%)$. Allowing the median incubation period to range from 7.5 to 12.3 years, the

30 estimate of the proportion diagnosed can vary from $77 \%$ to $84 \%$.

31 Discussion. The proportion of diagnosed HIV infections appears to have not yet reached $90 \%$

32 among Japanese nationals. Compared with the peak incidence from 2005-2008, new HIV

33 infections have clearly been in a declining trend; however, there are still more than 1,000 new

34 HIV infections per year in Japan. To increase the diagnosed proportion of HIV infections, it is

35 critical to identify people who have difficulty accessing consultation, testing, and care, and to

36 explore heterogeneous patterns of infection. 


\section{Introduction}

39 Following an infection with human immunodeficiency virus (HIV), development of acquired immunodeficiency syndrome (AIDS) takes about 10 years (Munoz et al., 1997). The long

41 incubation period makes it difficult to directly observe the incidence and prevalence of HIV infections over time. To offer insights into the epidemiology of HIV-infected and -incubating individuals over time, and to evaluate public health control programs, various statistical modeling approaches have been proposed to date (Brookmeyer \& Gail, 1994; Donnelly \& Cox, 2001; Jewell et al., 1992). Of these, a backcalculation method using a simple integral equation to model AIDS incidence as arising from the HIV incidence convoluted with the independently and identically distributed incubation period allows estimation of the HIV incidence based on epidemiological surveillance data (Brookmeyer \& Gail, 1986; Gail \& Brookmeyer, 1988). Assuming that the reported number of AIDS cases certainly and accurately captures the actual number of AIDS incidence in industrialized countries, the backcalculation method greatly improves our understanding of the epidemiology of HIV infection, attributing the observed AIDS data to HIV infection events as a function of time. Understanding the transmission dynamics of HIV using such statistical models is in line with the concept of treatment cascade, introduced by the Joint United Nations Programme on HIV/AIDS (UNAIDS). The so-called care cascade aims to identify and fill gaps in the continuum of services for testing, care, and effective treatment of HIV (UNAIDS, 2014). In relation to this, the UNAIDS report has led to the global initiative " $90-90-90$ " by 2020 that sets out goals in care cascades to achieve the following: $90 \%$ of people living with HIV know their HIV status, $90 \%$ of people diagnosed with HIV have access to antiretroviral therapy (ART), and 
$6090 \%$ of people receiving ART have suppressed viral loads (UNAIDS, 2014). UNAIDS even

61 aims to achieve 95-95-95 at a global level by the year 2030, contributing to successfully

62 controlling HIV and AIDS, as supported by the so-called test-and-treat strategy (Granich et al.,

63 2009; Granich et al., 2017). To quantify the situation of each country, monitoring diagnosed

64 individuals is essential; moreover, estimation of the diagnosed proportion of HIV infections must

65 be supported by firm scientific methods, to estimate the first part of the three 90-90-90 targets,

66 i.e., 90\% of HIV-infected people know their HIV status. In Japan, an analysis of blood donors

67 took place in 2017 , in which it was estimated that $85.6 \%$ of HIV-infected individuals, regardless

68 of nationality, were diagnosed (Iwamoto et al., 2017). Nevertheless, it is known that the analysis

69 of voluntary blood donation data is prone to sampling bias of donors owing to the tendency of

70 people with high risk to repeatedly undertake anonymous laboratory testing through the practice

71 of blood donation (Kihara et al., 2000), and moreover, an exclusion of repeaters can result in

72 underestimation of the prevalence, resulting in overestimation of the fraction diagnosed, even

73 though such screening of a large number of people is very costly. Considering the need to

74 achieve continued monitoring of the diagnosed proportion of HIV-infected individuals,

75 development of a reasonable yet scientifically rigorous method based on other datasets would be

76 beneficial, especially using epidemiological surveillance data (Hsieh et al., 2012; Cuadros \&

77 Abu-Raddad, 2016; Hsieh \& Lin, 2016; Mumtaz et al., 2018).

Despite the clear need for epidemiological estimation of the number of undiagnosed HIV

79 infections, the surveillance data in Japan possesses two technical problems. First, while the

80 definition of AIDS has remained nearly unchanged over time, reporting AIDS cases that were

81 previously diagnosed as HIV-infected cases has never been mandated (Nishiura, 2007). This

82 makes it impossible to directly apply the simplest convolution equation to the data because the 
83 backcalculation method requires the count of all AIDS cases over time. Surveillance in Japan has

84 only consistently counted (i) HIV infections without AIDS at the time of diagnosis and (ii) AIDS

85 cases without previous diagnosis of HIV infection. Second, ART has been widespread since

861997 and has continuously improved the prognosis of HIV infection. Explicit incorporation of

87 treatment requires us to account for not only the treatment coverage but also the treatment details

88 (e.g., details of combination therapy), adherence, and many other factors. While there are a

89 number of possible methods to address these issues, including those using CD4 data (e.g. van

90 Sighem et al., 2017) or molecular biomarkers, a simple yet tractable estimation method that rests

91 on epidemiological surveillance data and that can reasonably overcome these problems is called

92 for.

93 In the present study, the aim was to address the abovementioned issues, estimating the

94 HIV incidence among Japanese nationals, and also to offer statistical estimates of undiagnosed

95 HIV infections and the proportion of diagnosed HIV infections over time.

96 Materials \& Methods

97 Surveillance data of HIV and AIDS in Japan

98 The present study investigated the epidemiological surveillance data of HIV and AIDS in Japan,

99 which is publicly reported by the Committee of AIDS Trends (2018), belonging to the Ministry

100 of Health, Labor and Welfare, Japan. Of the reported datasets, our analyses are focused on

101 Japanese nationals because estimation of infection among foreigners requires accounting for

102 human migration, and the decision of migratory behavior (e.g. leaving Japan) is highly

103 dependent on the diagnosis of HIV infection and AIDS. As of the end of 2017, there were 16,663

104 HIV infections and 7,587 AIDS cases among Japanese nationals (Committee of AIDS Trends,

105 2018). As mentioned, HIV diagnoses reflect HIV-infected individuals who undertook voluntary 
106 diagnostic testing before the onset of AIDS. An AIDS case indicates a patient who has never

107 been diagnosed with HIV infection prior to an AIDS diagnosis and who meets the clinical

108 diagnostic criteria: (i) confirmed HIV infection and (ii) the presence of one of 23 indicator

109 diseases representing opportunistic infections or tumors. According to the Infectious Disease

110 Law, HIV and AIDS are classified as a category V notifiable disease, and once diagnosed,

111 physicians must notify the case within 7 days of diagnosis. In the present study, the yearly

112 incidence of HIV infections and AIDS diagnoses from 1985 to 2017 was used. The data are

113 structured by sex and also by the most likely route of transmission (e.g., heterosexual,

114 homosexual or intravenous drug use, based on a physician's interview of patients). The latter

115 information, i.e., the mode of transmission, is discarded because it is believed that a substantial

116 proportion of men having sex with men do not disclose the actual contact and inform physicians

117 that they acquired infection through heterosexual contact (Inoue et al., 2015). Thus, a stratified

118 estimation by sex was conducted. Although the magnitude of the epidemic in Japan is relatively

119 small compared with that in Western industrialized countries, the incidence of HIV infection in

120 Japan is believed to have steadily increased over time, especially among men who have sex with

121 men and young adults (Kihara et al., 2003; Nemoto, 2004).

\section{Derivation of likelihood using a mathematical model}

The proposed statistical model is derived from the following partial differential equation

124 (PDE) model, which is referred to as the McKendrick equation (Nishiura \& Inaba, 2011; Ejima

125 et al., 2014). Figure 1 shows a compartmental diagram of the data-generating process. Once

126 infected with HIV, individuals who are undiagnosed and in the incubation period experience two

127 different hazards, i.e., the force of HIV diagnosis $\alpha(t)$ that depends on calendar time $t$ and the

128 hazard of illness onset $\rho(\tau)$ that depends on the time elapsed since infection $\tau$. Let $h(t, \tau)$ be 
129 undiagnosed incubating HIV infections at calendar time $t$ and the time since infection $\tau$ (i.e.,

130 undiagnosed HIV infection without AIDS), the dynamics of HIV diagnosis and illness onset are

131 described by

$$
\left(\frac{\partial}{\partial t}+\frac{\partial}{\partial s}\right) h(t, s)=-(\alpha(t)+\rho(s)) h(t, s),
$$

133 with a boundary condition

$$
\lambda(t):=h(t, 0)
$$

135 where $\lambda(t)$ represents the HIV incidence (i.e., the number of new HIV infections) at calendar time

$136 t$. It should be noted that $\rho(\tau)$ yields $f(\tau)$, the probability density function of the incubation period

137 as follows:

$$
f(s)=\rho(s) \exp \left(-\int_{0}^{s} \rho(y) d y\right)
$$

139 for $s>0$. It is well known that the McKendrick equation can be solved along the characteristic

140 line, i.e.,

$$
h(t, s)=\lambda(t-s) \exp \left(-\int_{t-s}^{t} \alpha(x) d x-\int_{0}^{s} \rho(y) d y\right)
$$

142 for $t^{-} s>0$. Equations (1) and (4) indicate that the incidence of HIV diagnosis at calendar time $t$, $143 u(t)$, is written as

$$
\begin{aligned}
u(t) & =\int_{0}^{t} \alpha(t) h(t, s) d s \\
& =\int_{0}^{t} \alpha(t) \lambda(t-s) \exp \left(-\int_{t-s}^{t} \alpha(x) d x-\int_{0}^{s} \rho(y) d y\right) d s,
\end{aligned}
$$

146 and similarly, the incidence of AIDS cases at time $t, a(t)$, is

$$
a(t)=\int_{0}^{t} \rho(s) h(t, s) d s
$$


148

$$
=\int_{0}^{t} \rho(s) \lambda(t-s) \exp \left(-\int_{t-s}^{t} \alpha(x) d x-\int_{0}^{s} \rho(y) d y\right) d s .
$$

149 Equations (5) and (6) read similarly to the so-called extended backcalculation (Hall et al., 2008),

150 which is derived from a competing risk model (Marschner, 1994; Cui \& Becker, 2000). The

151 abovementioned process can be used as the generalization.

\section{Statistical model and estimation}

153 The datasets are reported in a discrete time interval (i.e., year); thus, here I discretized models (5)

154 and (6) as

$$
u_{t}=\sum_{s=1}^{t} \lambda_{t-s} \alpha_{t} \prod_{x=t-s-1}^{t-1}\left(1-\alpha_{x}\right) \prod_{y=1}^{s-1}\left(1-\rho_{y}\right)
$$

156 and

$$
a_{t}=\sum_{s=1}^{t} \lambda_{t-s} \rho_{s} \prod_{x=t-s-1}^{t-1}\left(1-\alpha_{x}\right) \prod_{y=1}^{s-1}\left(1-\rho_{y}\right) .
$$

158 There is no prior notion as to the shape of the epidemic curve (i.e. the frequency of transmission)

159 over time. Thus, the incidence of HIV infection in year $t, \lambda_{t}$, is modeled as a step function:

$$
\lambda_{t}= \begin{cases}\lambda_{1} & \text { for } t<1989 \\ \lambda_{2} & \text { for } 1989 \leq t<1993 \\ \vdots & \\ \lambda_{9} & \text { for } 2013 \leq t\end{cases}
$$

161 such that the yearly incidence can be directly dealt with as the parameter. The yearly probability

162 of diagnosis in year $t, \alpha_{t}$, is similarly modeled as

163

$$
\alpha_{t}= \begin{cases}\alpha_{1} & \text { for } t<1989 \\ \alpha_{2} & \text { for } 1989 \leq t<1993 \\ \vdots & \\ \alpha_{9} & \text { for } 2013 \leq t\end{cases}
$$


164 The probability mass function of the incubation period is assumed as known, and in discrete

165 time, this is written as $\rho_{s} \prod_{y=1}^{s-1}\left(1-\rho_{y}\right)$. As is widely assumed for HIV infection, the incubation

166 period is modeled using the Weibull distribution. Using the property of Weibull distribution with

167 the scale parameter $\eta$ and shape parameter $k$, the discrete Weibull model is connected to the

168 continuous version as

169

$$
\rho_{s}=1-\frac{\exp \left(-\left(\frac{t+1}{\eta}\right) k\right)}{\exp \left(-\left(\frac{t}{\eta}\right)^{k}\right)}
$$

170 and

$$
\prod_{y=1}^{t-1}\left(1-\rho_{y}\right)=\exp \left(-\left(\frac{t}{\eta}\right) k\right)
$$
computed as

$$
x_{t}=\sum_{s=1}^{t} \lambda_{t-s} \prod_{x=t-s-1}^{t-1}\left(1-\alpha_{x}\right) \prod_{y=1}^{s-1}\left(1-\rho_{y}\right)
$$

175 The diagnosed proportion of HIV infections is calculated either as $\sum(a+u) / \sum(x+a+u)$ or $\sum u$ /

$176 \sum(x+u)$, taking the summations over time. The former calculates the proportion of diagnosed

177 HIV-positive individuals out of the cumulative number of HIV-positive individuals. This

178 calculation has the drawback of including patients with AIDS who have already died by the year

179 of calculation. As of 2017, it has been reported that a total of 2,321 cases resulted in death

180 (Iwamoto et al., 2017). Alternatively, the latter calculates the fraction of individuals who are HIV

181 positive but have not yet developed AIDS out of the cumulative number of HIV-positive

182 individuals but including undiagnosed individuals, considering that the incubation period in most 
183 cases of HIV infection is now considerably extended by ART. The drawback of the latter

184 calculation is that patients with AIDS who have survived and have received ART are excluded;

185 thus, the calculated proportion may not be strictly in line with the target figure in the first goal of

186 the 90-90-90 initiative. Therefore, when estimating the undiagnosed number of HIV infections

187 and the diagnosed proportion at the end of 2017, both calculations are made, and the former is

188 adjusted by subtracting 2,321 AIDS deaths from the cumulative count of AIDS cases.

189 To quantify the proposed system of equations, we estimate parameters $\lambda_{\mathrm{t}}$ and $\alpha_{\mathrm{t}}$ by means

190 of the maximum likelihood method. Considering that HIV infections are generated as the

191 nonhomogenous Poisson process, the resulting HIV diagnoses and AIDS cases would also

192 follow Poisson distributions. The likelihood function of HIV diagnoses is

$$
L_{1}=\text { constant } \times \prod_{t=1985}^{2017} E\left(u_{t}\right)^{r}{ }_{t} \exp \left(-E\left(u_{t}\right)\right),
$$

194 where $r_{\mathrm{t}}$ denotes the reported (observed) number of HIV diagnoses in year $t$ in the surveillance record. Similarly, the likelihood of new AIDS diagnoses is

$$
L_{2}=\text { constant } \times \prod_{t=1985}^{2017} E\left(a_{t}\right)^{w} t \exp \left(-E\left(a_{t}\right)\right),
$$

where $w_{\mathrm{t}}$ denotes the reported number of new AIDS diagnoses in year $t$. Consequently, the total likelihood $L$ is given by

$$
L=L_{1} L_{2}
$$

Maximum likelihood estimates of parameters are obtained by minimizing the negative logarithm

201 of Eq. (16). As mentioned above, the incubation period distribution is assumed as known, and to

202 address the uncertainty, three different estimates are derived from published studies (Boldson et

203 al., 1988; Brookmeyer \& Goedert, 1989; Munoz \& Xu, 1996). A widely cited estimate by

204 Brookmeyer \& Goedert (1989) was derived from the study of patients with hemophilia over 20 
205 years of age with $\eta=11.6$ and $k=2.5$, resulting in a median incubation period of 10.0 years.

206 Boldson et al. (1988) investigated a cohort of AIDS cases in San Francisco with $\eta=14.3$ and

$207 k=2.5$, yielding a median incubation period of 12.3 years. The estimate by Munoz and Xu (1996)

208 was obtained from the Multicenter AIDS Cohort Study with $\eta=10.0$ and $k=1.3$, and the median

209 incubation period is 7.5 years. All three estimates have been used in the present study to address

210 uncertainty with respect to the incubation period. In addition to (14) and (15), we have also

211 explored the over-dispersed likelihood function, employing the negative binomial distribution

212 with time-independent dispersion parameter for HIV and AIDS counts, respectively, (Althaus,

213 2015) and compared the Akaike Information Criterion (AIC) against Poisson distributed

214 likelihood, as part of sensitivity analysis.

215 The $95 \%$ confidence interval (CI) of parameters was derived from the profile likelihood.

216 The 95\% CI of model estimates (e.g., the number of undiagnosed HIV infections and the

217 proportion diagnosed) was derived using a parametric bootstrap method. In the bootstrapping

218 exercise, model parameters were resampled from a multivariate normal distribution with vectors

219 of mean $\boldsymbol{\theta}$ and standard deviation $\boldsymbol{\sigma}$. The latter vector was derived from the covariance matrix,

220 taking diagonal elements of the inverse Hessian matrix $\left(\sigma^{2}=\operatorname{diag}\left(H^{-1}(\theta)\right)\right)$. For each set of

221 parameters, the model solution is obtained, and 1,000 times of parameter resampling results in a

222 simulated distribution of model solutions. By taking the 2.5th and 97.5th percentile points of the

223 simulated distribution, the $95 \% \mathrm{CI}$ is obtained. All statistical data were analyzed using R version

2243.1 (Comprehensive R Archive Network) (R Core Team, 2016) and JMP version 12.0.1

225 statistical software (SAS Institute Inc., Cary, NC, USA). 


\section{Ethical considerations}

227 In the present study, the analyzed data are publicly available (Committee of AIDS Trends, 2018).

228 As such, the datasets used in our study are deidentified and fully anonymized in advance, and the

229 analysis of publicly available data with no identifying information does not require ethical

230 approval.

\section{Results}

232 Estimated parameters, i.e., yearly incidence and yearly probability of diagnosis, are

233 shown in Fig. 2. With the assumed median incubation period of 10.0 years, the yearly incidence

234 was the highest from 2005-2008, with an estimated 1,972 (95\% CI: 1,829, 2,115) infections per

235 year (Fig. 2A). Subsequently, the incidence began to decline; the yearly estimate in the most

236 recent interval (from 2013-2017) was 1,179 (95\% CI: 1,047, 1,293) infections. The yearly

237 probability of diagnosis has monotonously improved over time (Fig. 2B). The estimated

238 diagnosis probability by 1999 was less than 10\%, but the latest estimate from 2013-2017 was

239 15.6\% (95\% CI: 14.8\%, 16.4\%). The qualitative patterns of HIV incidence and diagnosis did not

240 vary greatly, even when shorter and longer median incubation periods were used (Figs. 2C and

241 2D). Figures 2E and 2F show maximum likelihood estimates of the incidence and probability of

242 diagnosis by sex. The incidence in males was the highest from 2005-2008; the latest estimate

243 from 2013-2017 ranged from 1,015 to 1,363 infections per year, assuming a median incubation

244 period from 7.5 to 12.3 years. Similarly, the incidence in females was highest from 1993-1996,

245 ranging from 86 to 97 infections per year; the latest yearly incidence ranged from 31 to 54

246 infections with a median incubation period of 7.5 to 12.3 years. The yearly probability of

247 diagnosis among males behaved similarly to that of the entire population, but there was no

248 apparent improvement in the frequency of diagnosis among females. In general, female enjoyed 
249 higher rate of diagnosis than male. For the entire population with median incubation period at

25010.0 years, AIC with the Poisson distributed likelihood was 650.7, while that with negative

251 binomially distributed likelihood was 655.0 , indicating that the dataset was not over-dispersed.

252 In fact, dispersion parameters for HIV and AIDS were estimated at greater than 100, indicating

253 that Poisson distribution has sufficiently captured the variation.

254 Figure 3 shows a comparison between the observed and predicted number of HIV

255 diagnoses and AIDS cases. All three models with different median incubation periods yielded

256 almost identically good fit to the data (Fig. 3A), reflecting mutual compensations between $\lambda$ and

$257 \alpha$ to fit to the data. Even though the number of diagnosed HIV infections and AIDS cases was

258 relatively small for females, the proposed model successfully captured the observed patterns of

259 HIV diagnoses and AIDS cases by sex (Fig. 3B).

260 Figure 4 shows the estimated undiagnosed number of HIV infections and the estimated

261 proportion of diagnosed HIV-positive individuals over time, among Japanese nationals. Using

262 the median incubation period of 10.0 years (Fig. 4A), undiagnosed HIV infection was estimated

263 to have peaked in 2009 with 7,532 (95\% CI: 6,911, 8,152) infections. In the latest time interval,

264 from 2013-2017, it was estimated that 5,363 (95\% CI: 4,809, 5,917) infections remained

265 unrecognized. Varying the median incubation period from 7.5 to 12.3 years, the maximum

266 likelihood estimate of undiagnosed HIV infections in the latest time interval ranged from 4,041

267 to 6,552 infections. These findings indicate that the cumulative number of HIV infections by the

268 end of 2017 was 29,613 (95\% CI: 29,059, 30,167) Japanese nationals, using the median

269 incubation period of 10.0 years, and can range from 28,291 to 30,802 individuals.

270 Including and excluding AIDS cases, the estimated proportions of diagnosed HIV

271 infections are shown in Figs. 4C and 4D. Including AIDS cases, the diagnosed proportion was 
272 estimated at $81.9 \%$ (range $78.7 \%$ to $85.7 \%$ ) using the median incubation period of 10.0 (7.5 to

27312.3 ) years. Excluding AIDS cases, the estimate was $75.7 \%$ (range $71.8 \%$ to $80.5 \%$ ). Figures $4 \mathrm{E}$

274 and $4 \mathrm{~F}$ show the estimated number of undiagnosed HIV infections and the diagnosed proportion

275 by sex, excluding AIDS cases. Estimates of undiagnosed HIV infections among males behaved

276 similarly to the entire population of Japanese nationals, whereas those of females peaked in the

277 year 2001. In the latest time interval (2013-2017), it was estimated that 5,150 infections (range

2783,881 to 6,287 ) in males and 210 infections (range 162 to 255) in females remained

279 unrecognized, using the median incubation period of 10.0 (with the range of 7.5 to 12.3 ) years.

280 The diagnosed proportion of both males and females increased with time, and females tended to

281 yield higher estimates than males. In the latest time interval from 2013-2017, the diagnosed

282 proportion (excluding AIDS cases) was estimated at $75.3 \%$ (range $71.4 \%$ to $80.2 \%$ ) among

283 males and $82.1 \%$ (range $79.1 \%$ to $85.6 \%$ ) among females.

284 Figure 5 shows the undiagnosed number of HIV infections and the proportion of 285 diagnosed infections at the end of 2017. The uncertainty bound was greatest with an assumed 286 median incubation period of 12.3 years, with an estimated 6,552 infections (95\% CI: 5,632, 287 7,471). Figure 5B shows the diagnosed proportion, including and excluding AIDS cases, with $28895 \%$ confidence intervals. Even when AIDS cases were included, the 2,321 deaths known up to 289 that point were subtracted from AIDS cases in advance of the calculation. Assuming that the 290 median incubation period was 10.0 years, the calculation, inclusive of surviving AIDS cases, 291 yielded 80.3\% (95\% CI: 78.7\%, 82.0\%); when excluding AIDS cases, the proportion was $75.7 \%$ 292 (95\% CI: 73.8\%, 77.6\%). 


\section{Discussion}

294 The present study estimated the incidence and diagnosed fraction of HIV infections among

295 Japanese nationals, devising an original model that captures the data generating process of HIV

296 and AIDS in the epidemiological surveillance. By the end of 2017, the cumulative number of

297 HIV infections was estimated to be about 30,000 cases, of which 4,000 to 6,000 were considered

298 to have remained undiagnosed. Assuming that the median incubation period was 10.0 years, $80 \%$

299 of infections have ever been diagnosed; accounting for the uncertainty in a median incubation

300 period ranging from 7.5 to 12.3 years, the estimate of the diagnosed proportion can range from

$30177 \%$ to $84 \%$. To the author's knowledge, the present study is the first to offer firm statistical

302 estimates of the incidence and diagnosed proportion of HIV infections based on epidemiological

303 surveillance data in Japan, using an explicit mathematical modeling approach.

304 There are two take-home messages from the results of this study. First, regardless of

305 whether AIDS cases are included, the proportion of diagnosed HIV infections appears not to

306 have reached $90 \%$ among Japanese nationals. Although some estimates exceed $80 \%$, even after

307 subtraction of known deaths owing to AIDS, the findings echo those of a published study that

308 analyzed blood donor data (Iwamoto et al., 2017). The published blood donor-based estimate

309 indicated that $85.6 \%$ have been diagnosed at the end of 2015 , which was prone to sampling bias

310 with substantial potential of both over- and underestimation, and the present study validated that

311 the surveillance-based estimate was slightly below and not too far from the published figure.

312 These findings pose a critical problem in Japan for controlling HIV and AIDS. In the present

313 study, the rate of diagnosis was shown to have improved with time, and the trend was

314 particularly apparent among men, mainly comprising men having sex with men (MSM). The

315 findings of the present study indicate that there would be a certain number of infected individuals 
316 who may not have proper access to consultation, testing, and care with privacy protection. To

317 identify the attributes of such HIV-infected individuals in greater detail, the investigation must

318 be extended to explore heterogeneous patterns, including age-dependence, spatial heterogeneity,

319 and other background characteristics. These are my ongoing research interests.

320 Second, compared with the peak from 2005-2008, the incidence showed a declining

321 trend. Compared with the estimate in 2005-2008, the upper 95\% CIs of the next two time

322 periods (2009-2012 and 2013-2017) were significantly lower than those in the peak period. In

323 fact, a declining trend has also been seen in other datasets, including the incidence of counseling

324 and blood testing at local health centers and the proportion of HIV-positive blood donors over

325 time (Committee of AIDS Trends, 2018). The present study results support that these observed

326 declines are partially attributable to actual decreases in the incidence of HIV infection in Japan.

327 The underlying mechanisms of such decreases have yet to be explored using a mathematical

328 model, perhaps requiring modeling of the saturated effect (Heesterbeek \& Metz, 1993) together

329 with statistical estimates of the effective reproduction number (Kretzschmar et al., 2013). The

330 success of Japan's controlling HIV transmission among the core population, i.e. MSM, reflecting

331 the set-up of gay community centers and scale-up of gay non-governmental organizations'

332 activities (Sherriff et al., 2017), could potentially be objectively demonstrated in such an

333 analysis. In addition, it must be remembered that the yearly incidence still remains above 1,000

334 infections; moreover, such a declining trend is not evident among females, although the rate of

335 diagnosis in female has steadily been higher than that in male. Higher frequency of diagnosis

336 among female than male might reflect better awareness of the risk that results from heterosexual

337 transmission (e.g. through foreign partner's diagnosis). 
Although the present study was motivated by the need for quantifying the care cascade in

339 Japan, in accordance with the goals of 90-90-90, a few technical issues must be noted to interpret

340 the estimates and apply the present results to the evaluation. First, Japanese estimates of the latter

341 two goals of the 90-90-90 initiative, i.e., access to ART and virus suppression, rest on

342 questionnaire surveys conducted in the prefectures, which do not distinguish between infected

343 individuals who are Japanese nationals and those who are not (Iwamoto et al., 2017). Thus, our

344 estimates of the diagnosed proportion of HIV infections among Japanese nationals alone cannot

345 immediately be compared with subsequent existing proportions as if they were sampled from the

346 same population. Whereas estimation of the HIV incidence among non-Japanese nationals is an

347 ongoing research subject, it is frequently the case that infection with HIV or illness onset of

348 AIDS acts as a trigger for foreigners to leave the country; therefore, incorporation of their

349 involvement in the transmission dynamics of Japan requires that very careful attention be paid to

350 migration events (Matsuyama et al., 2018; Sakamoto et al., 2018; Yuan and Nishiura, 2018), and

351 ideally, that information is supported by individual-based data. Second, the clinical definition of

352 AIDS in Japan depends on indicator diseases, imposing a certain extent of uncertainty in

353 diagnosis. For instance, Japan has a number of designated AIDS Core Hospitals, and HIV

354 diagnoses in those institutes involve screening of common opportunistic infections upon

355 diagnosis of HIV infection, which sensitively leads to the diagnosis of AIDS. Compared with

356 HIV-infected individuals diagnosed at local health centers, the frequency of AIDS diagnosis may

357 be higher in the designated hospitals, calling for the validation of estimates using other methods.

358 Using other datasets including biomarkers or CD4 count data can act as another potential work to

359 be built on this study. Third, in the present study, we struggled with subtraction of AIDS deaths

360 from the calculation of the diagnosed proportion of infections; this problem essentially stems 
361 from the absence of a case registration system in Japan. Once diagnosed, infected individuals are

362 never longitudinally monitored by the government, considerably complicating prevalence

363 estimation. With a registration system of HIV-infected individuals, statistical monitoring of the

364 second and third goals of 90-90-90 can be achieved in real time and in a very reasonable manner.

365 Five technical limitations must be noted. First, the present study did not account for

366 uncertainties other than variations in length of the incubation period. There has been a concern

367 that the incubation period has probably shortened over time (Nakamura et al., 2011), but I did

368 not have substantial data to support this issue. Second, the natural history of HIV infection has

369 yet to be explored in-depth; an explicit proportion of HIV individuals who never develop AIDS

370 over the course of infection is missing. Third, other than sex, the present study accepted

371 homogeneity in the natural course and diagnosis of infection. Our future studies will address

372 several heterogeneities. Fourth, estimates rested on yearly data, and the precision was limited

373 (e.g., with use of the step function for every 4 years). The use of smoothing with nonparametric

374 back-projection is another of our ongoing studies (Becker, 1997). Fifth, the present study focused

375 on the incidence estimation, and more explicit modeling of the transmission dynamics, including

376 those highlighting the role of MSM (Yamamoto et al., 2018), is the subject for future studies.

377 Despite these limitations, the present study successfully estimated the incidence of HIV

378 infections, undiagnosed number of infections, and the proportion diagnosed in real time, using

379 limited but readily available epidemiological surveillance data. Improved estimates using age

380 and geographical data, as well as estimates based on other methods, are to follow, which will

381 boost studies of epidemiological estimation in this area in Japan. 


\section{Conclusions}

383 In the present study, a statistical modeling method was developed for the estimation of HIV

384 incidence in Japan and estimates made of the undiagnosed number of HIV infections and the

385 proportion of diagnosed HIV infections over time. Using the McKendrick equation, a general

386 convolution-like equation was derived, allowing for joint estimation of the HIV incidence and

387 time-dependent rate of diagnosis. By the end of 2017, the cumulative number of HIV infections

388 was estimated to be about 30,000 , and about $80 \%$ of infections have ever been diagnosed.

389 Accounting for the uncertainty in the median incubation period ranging from 7.5 to 12.3 years,

390 estimates of the diagnosed proportion of HIV infections can range from $77 \%$ to $84 \%$. The

391 proportion of diagnosed HIV infections appears not to have reached $90 \%$ among Japanese

392 nationals.

393

394 Acknowledgment

395 We thank Analisa Avila, ELS, of Edanz Group for editing a draft of this manuscript.

397 References

398 Althaus C. 2015. Ebola superspreading. Lancet Infect Dis 15:507-508.

399 Becker NG. 1997. Uses of the EM algorithm in the analysis of data on HIV/AIDS and other

$400 \quad$ infectious diseases. Statistical Methods in Medical Research 6:24-37.

401 Boldson JL, Jensen JL, Sogarrd J, Sorensen M. 1988. On the incubation time distribution and 402 the Danish AIDS data. Journal of the Royal Statistical Society, Series A 151:42-43. 
403 Brookmeyer R, Gail MH. 1986. Minimum size of the acquired immunodeficiency syndrome 404 (AIDS) epidemic in the United States. Lancet 2:1320-1322.

405 Brookmeyer R, Gail MH. 1994. AIDS Epidemiology: A Quantitative Approach. New York: $406 \quad$ Oxford University Press.

407 Brookmeyer R, Goedert JJ. 1989. Censoring in an epidemic with an application to 408 haemophilia-associated AIDS. Biometrics 45:325-335.

409 Committee of AIDS Trends, Ministry of Health, Labor and Welfare, Japan. 2018. Annual 410 Report of the Trend of HIV and AIDS in Japan. Tokyo: Ministry of Health, Labor and 411 Welfare. Available at: http://api-net.jfap.or.jp/status/(accessed 10 October 2018).

412 Cuadros DF, Abu-Raddad LJ. 2016. Geographical Patterns of HIV Sero-Discordancy in High 413 HIV Prevalence Countries in Sub-Saharan Africa. International Journal of 414 Environmental Research and Public Health 13:865. DOI: 10.3390/ijerph13090865.

415 Cui JS, Becker NG. 2000. Estimating HIV incidence using dates of both HIV and AIDS 416 diagnoses. Statistics in Medicine 19:1165-1177.

417 Donnelly CA, Cox DR. 2001. Mathematical biology and medical statistics: contributions to 418 the understanding of AIDS epidemiology. Statistical Methods in Medical Research $419 \quad 10: 141-154$.

420 Ejima K, Aihara K, Nishiura H. 2014. Probabilistic differential diagnosis of Middle East 421 respiratory syndrome (MERS) using the time from immigration to illness onset among 422 imported cases. Journal of Theoretical Biology 346:47-53.

423 Gail MH, Brookmeyer R. 1988. Methods for projecting course of acquired 424 immunodeficiency syndrome epidemic. Journal of the National Cancer Institute 80:900425 911. 
426 Granich RM, Gilks CF, Dye C, De Cock KM, Williams BG. 2009. Universal voluntary HIV testing with immediate antiretroviral therapy as a strategy for elimination of HIV transmission: a mathematical model. Lancet 373:48-57. and feasible. Lancet 390:341-343.

Heesterbeek JAP, Metz J. 1993. The saturating contact rate in marriage-and epidemic Taiwan: A Modeling Analysis. Current HIV Research 14:138-147. models. Journal of Mathematical Biology 31:529-539.

Hsieh YH, Lin PC. 2016. Current Trends and Future Projection of HIV/AIDS Epidemic in

Hsieh YH, Ruan Y, Chen CW, Shi W, Li D, Luo F, Shao Y. 2012. HIV prevalence and underreporting of men who have sex with men in Beijing. International Journal of STD \& AIDS 23:606-607.

Inoue Y, Takaku Y, Yajima T, Ikushima Y. 2015. A survey comparing the route of HIV transmission reported by patients to their physicians and the self-reported "actual route". Nihon Koshu Eisei Zasshi 62:106-116 (in Japanese).

Iwamoto A, Taira R, Yokomaku Y, Koibuchi T, Rahman M, Izumi Y, Tadokoro K. 2017. The HIV care cascade: Japanese perspectives. PLoS One 12:e174360.

Jewell NP, Dietz K, Farewell VT. 1992. AIDS Epidemiology: Methodological Issues. Berlin, Birkhäuser.

Kihara M, Imai M, Shimizu M. 2000. Trends of HIV infections among blood donors. Infectious Agents Surveillance Report 21:140-141 (in Japanese). 
447

448

449

450

451

452

453

454

455

456

457

458

459

460

461

462

463

464

465

466

467

468

469

Kihara M, Ono-Kihara M, Feldman MD, Ichikawa S, Hashimoto S, Eboshida A, Yamamoto T, Kamakura M. 2003. HIV/AIDS surveillance in Japan, 1984-2000. Journal of Acquired Immune Deficiency Syndromes 32:S55-S62.

Kretzschmar ME, Schim van der Loeff MF, Birrell PJ, De Angelis D, Coutinho RA. 2013. Prospects of elimination of HIV with test-and-treat strategy. Proceedings of the National Academy of Sciences of the United States of America 110:15538-15543.

Marschner IC. 1994. Using time of first positive HIV test and other auxiliary data in back - projection of AIDS incidence. Statistics in Medicine 13:1959-1974.

Matsuyama R, Akhmetzhanov AR, Endo A, Lee H, Yamaguchi T, Tsuzuki S, Nishiura H. 2018. Uncertainty and sensitivity analysis of the basic reproduction number of diphtheria: A case study of Rohingya refugee camp in Bangladesh, November-December 2017. PeerJ 6:e4583.

Mumtaz GR, Awad SF, Feizzadeh A, Weiss HA, Abu-Raddad LJ. 2018. HIV incidence among people who inject drugs in the Middle East and North Africa: mathematical modelling analysis. Journal of the International AIDS Society 21:e25102.

Muñoz A, Sabin CA, Phillips AN. 1997. The incubation period of AIDS. AIDS 11:S69-S76. Munoz A, Xu J. 1996. Models for the incubation of AIDS and variations according to age and period. Statistics in Medicine 15:2459-2473.

Nakamura H, Teruya K, Takano M, Tsukada K, Tanuma J, Yazaki H, Honda H, Honda M, Gatanaga H, Kikuchi Y, Oka S. 2011. Clinical Symptoms and Courses of Primary HIV-1 Infection in Recent Years in Japan. Internal Medicine 50:95-101.

Nemoto T. 2004. HIV/AIDS surveillance and prevention studies in Japan: summary and recommendations. AIDS Education and Prevention 16:27-42. 
470 Nishiura H. 2007. Lessons from previous predictions of HIV/AIDS in the United States and

471 Japan: epidemiologic models and policy formulation. Epidemiologic Perspectives and $472 \quad$ Innovations $4: 3$.

473 Nishiura H, Inaba H. 2011. Estimation of the incubation period of influenza A (H1N1-2009)

474 among imported cases: addressing censoring using outbreak data at the origin of

475 importation. Journal of Theoretical Biology 272:123-130.

476 R Core Team. 2016. The R Project for Statistical Computing. Vienna, Austria. Available at: https://www.R-project.org/ (accessed 11 October 2018).

478 Sakamoto Y, Yamaguchi T, Yamamoto N, Nishiura H. 2018. Modeling the elevated risk of yellow fever among travelers visiting Brazil, 2018. Theoretical Biology and Medical Modelling 15(1):9.

481 Sherriff N, Koerner J, Kaneko N, Shiono S, Takaku M, Boseley R, Ichikawa S. 2017.

482 Everywhere in Japan: an international approach to working with commercial gay businesses in HIV prevention. Health Promot Int. 32(3):522-534.

UNAIDS. 2014. 90-90-90: an ambitious treatment target to help end the AIDS epidemic. UNAIDS: Geneva. Available at: http://www.unaids.org/en/resources/documents/2014/90-90-90 (accessed 11 October 2018 ). Surveillance And Dublin Declaration Monitoring Networks. 2017. Reduction in undiagnosed HIV infection in the European Union/European Economic Area, 2012 to 2016. Eurosurveillance 22: doi: 10.2807/1560-7917.ES.2017.22.48.17-00771. 
492

494

495

496

497

498

499

500

501

502

503

504

505

506

507

508

509

510

511

512

513
Yamamoto N, Ejima K, Nishiura H. 2018. Modelling the impact of correlations between condom use and sexual contact pattern on the dynamics of sexually transmitted infections. Theoretical Biology and Medical Modelling 15: 6.

Yuan B, Nishiura H. 2018. Estimating the actual importation risk of dengue virus infection among Japanese travelers. PLOS ONE 13(6):e0198734

\section{Figure legends}

\section{Figure 1. Data-generating process of HIV infections and AIDS cases in Japan}

New HIV infections occur at rate $\lambda(t)$. While going undiagnosed as $h(t, \tau)$, there would be an increase in the time since infection $\tau$. Diagnosis of HIV takes place at a time-dependent rate $\alpha(t)$, and AIDS illness onset occurs at rate $\rho(\tau)$, which depends on the time since infection. Newly diagnosed HIV infections, and AIDS cases that had not been previously diagnosed with HIV, were notified to the surveillance system.

\section{Figure 2. Estimated HIV incidence and rate of diagnosis in Japan.}

A. The yearly incidence of HIV infection, assuming that the median incubation period is 10.0 years. The step function for every 4 years was used to model the incidence. The $95 \%$ confidence intervals were derived from profile likelihood. B. The yearly rate of diagnosis of HIV infection, assuming that the median incubation period is 10.0 years. C. Maximum likelihood estimates of the yearly incidence with different median incubation periods: $7.5,10.0$, and 12.3 years. D.

Maximum likelihood estimates of the yearly rate of diagnosis with different median incubation periods: $7.5,10.0$, and 12.3 years. E. Yearly incidence estimates by sex and different median 
514 incubation periods. Maximum likelihood estimates are shown. Note that a common logarithmic

515 scale is used on the vertical axis, to ease comparisons. F. Yearly rate of diagnosis estimates by

516 sex using different median incubation periods. Unfilled marks are for female, while filled marks

517 are for male. Maximum likelihood estimates are shown.

518 Figure 3. HIV diagnoses and AIDS cases in Japan, 1985-2017.

519 A. Comparisons between observed and predicted yearly number of HIV diagnoses and AIDS

520 cases. Different median incubation periods (i.e., 7.5, 10.0, and 12.3 years) were assumed, but

521 predicted values are mostly overlapped. B. Comparisons between observed and predicted values

522 by sex. Circles represent the observed number of HIV diagnoses whereas triangles represent that

523 of AIDS cases. Solid marks represent males; empty marks represent females. A common

524 logarithmic scale is used on the vertical axis. In both panels, bold grey lines represent lower and

525 upper $95 \%$ confidence intervals with the median incubation period of 10.0 years based on the

526 parametric bootstrap method.

527 Figure 4. Undiagnosed number and proportion of HIV infections in Japan, 1986-2017.

528 A. Estimates of undiagnosed HIV infections, assuming that the median incubation period is 10.0

529 years. The $95 \%$ confidence intervals were derived from profile likelihood. B. Maximum

530 likelihood estimates of undiagnosed HIV infections with different median incubation periods:

$5317.5,10.0$, and 12.3 years. C. Proportion of diagnosed infections out of the cumulative number of

532 HIV infections, inclusive of AIDS cases. D. Proportion of diagnosed infections out of the

533 cumulative number of HIV infections, excluding AIDS cases. E. Maximum likelihood estimates

534 of undiagnosed HIV infections by sex, with different median incubation periods: $7.5,10.0$, and

53512.3 years. Note that common logarithmic scale is used on the vertical axis. D. Proportion of 
536 diagnosed infections out of the cumulative number of HIV infections, excluding AIDS cases, by

537 sex.

538 Figure 5. Estimated undiagnosed HIV infections and proportion of diagnosed infections at

539 the end of 2017.

540 A. Estimates of undiagnosed HIV infections with different incubation periods. Whiskers extend

541 to lower and upper $95 \%$ confidence intervals derived using a parametric bootstrapping method.

542 B. Proportion of diagnosed infections out of the cumulative number of HIV infections, excluding

543 AIDS cases (solid circles) or including AIDS cases but subtracting 2,321 deaths (empty circles).

544 Whiskers extend to lower and upper $95 \%$ confidence intervals derived using a parametric

545 bootstrapping method.

546 


\section{Figure 1}

Data-generating process of HIV infections and AIDS cases in Japan

New HIV infections occur at rate $\lambda(t)$. While going undiagnosed as $h(t, \tau)$, there would be an increase in the time since infection $\tau$. Diagnosis of HIV takes place at a time-dependent rate $\alpha(t)$, and AIDS illness onset occurs at rate $\rho(\tau)$, which depends on the time since infection. Newly diagnosed HIV infections, and AIDS cases that had not been previously diagnosed with HIV, were notified to the surveillance system.

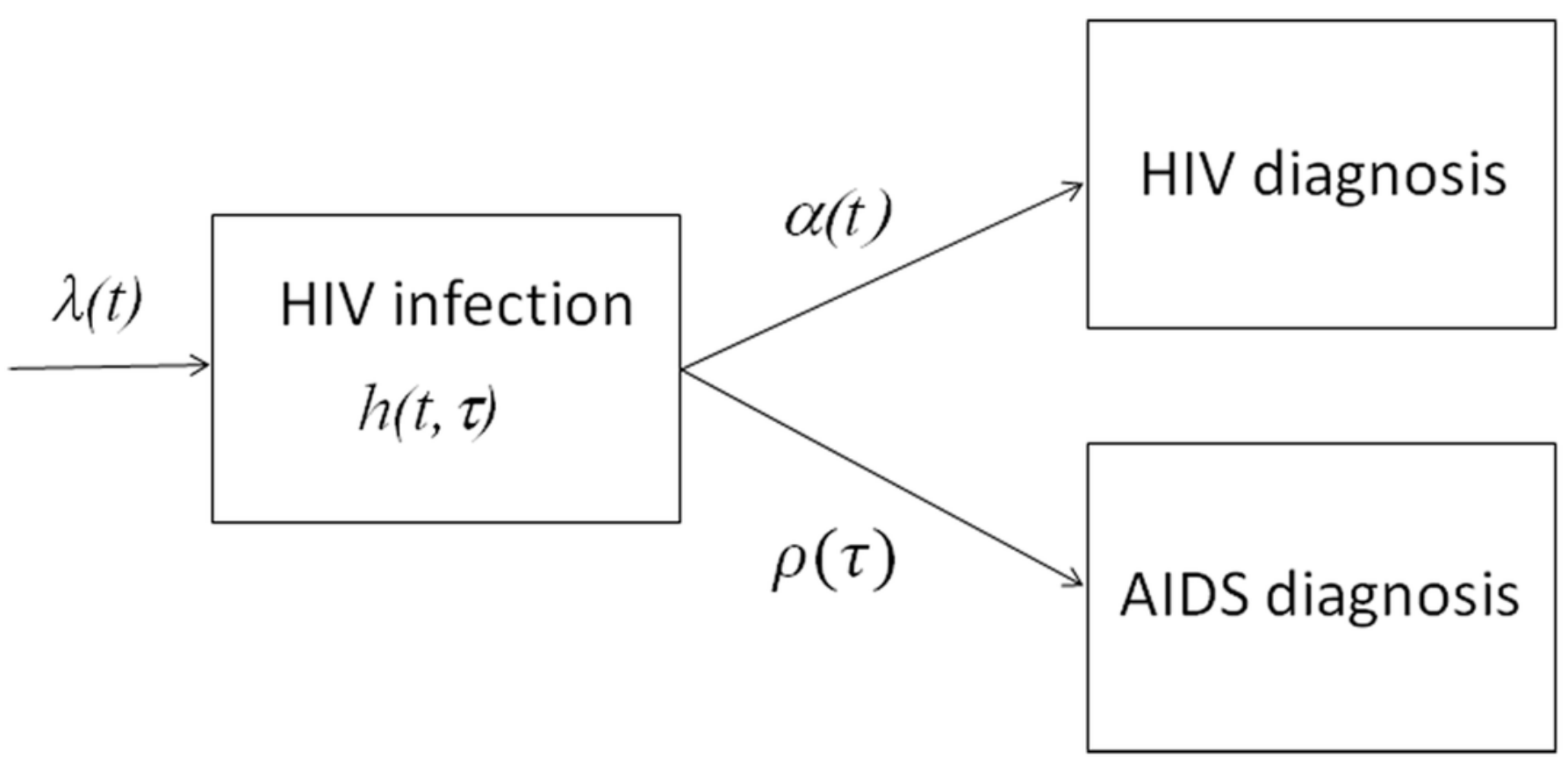




\section{Figure 2}

Estimated HIV incidence and rate of diagnosis in Japan.

A. The yearly incidence of HIV infection, assuming that the median incubation period is 10.0 years. The step function for every 4 years was used to model the incidence. The $95 \%$ confidence intervals were derived from profile likelihood. B. The yearly rate of diagnosis of HIV infection, assuming that the median incubation period is 10.0 years. C. Maximum likelihood estimates of the yearly incidence with different median incubation periods: 7.5, 10.0, and 12.3 years. D. Maximum likelihood estimates of the yearly rate of diagnosis with different median incubation periods: 7.5, 10.0, and 12.3 years. E. Yearly incidence estimates by sex and different median incubation periods. Maximum likelihood estimates are shown. Note that a common logarithmic scale is used on the vertical axis, to ease comparisons. $F$. Yearly rate of diagnosis estimates by sex and different median incubation periods. Maximum likelihood estimates are shown. 

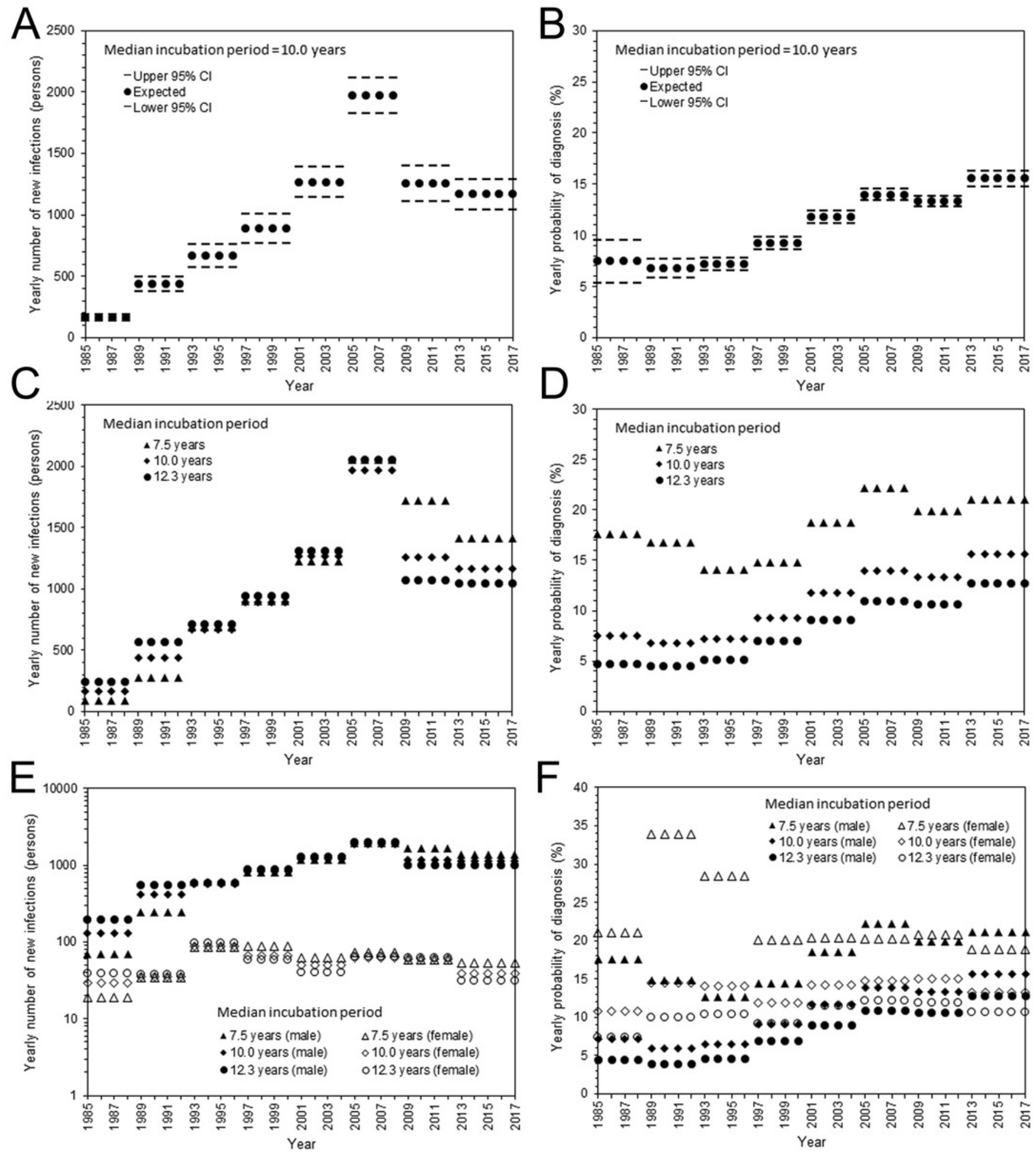
Figure 3

HIV diagnoses and AIDS cases in Japan, 1985-2017.

A. Comparisons between observed and predicted yearly number of HIV diagnoses and AIDS cases. Different median incubation periods (i.e., 7.5, 10.0, and 12.3 years) were assumed, but predicted values are mostly overlapped. B. Comparisons between observed and predicted values by sex. Circles represent the observed number of HIV diagnoses whereas triangles represent that of AIDS cases. Solid marks represent males; empty marks represent females. A common logarithmic scale is used on the vertical axis. In both panels, bold grey lines represent lower and upper 95\% confidence intervals with the median incubation period of 10.0 years based on the parametric bootstrap method.
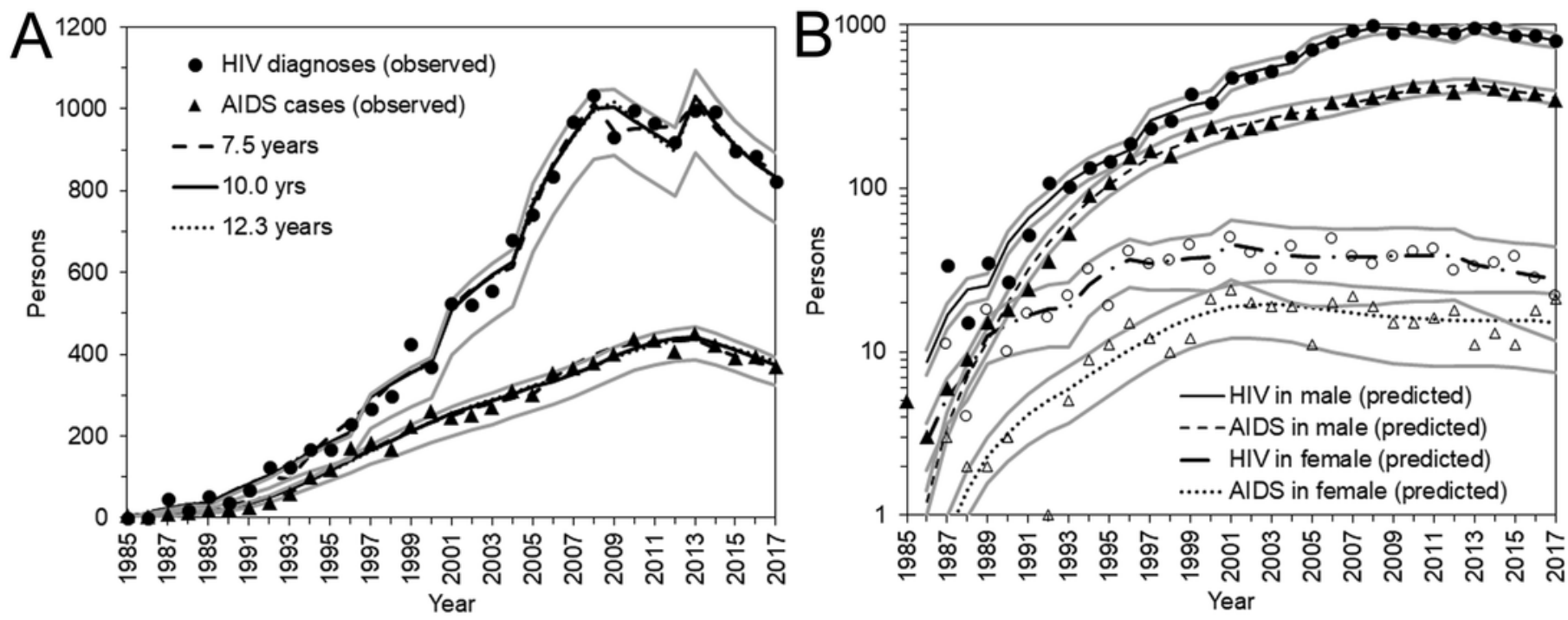


\section{Figure 4}

Undiagnosed number and proportion of HIV infections in Japan, 1986-2017.

A. Estimates of undiagnosed HIV infections, assuming that the median incubation period is 10.0 years. The $95 \%$ confidence intervals were derived from profile likelihood. B. Maximum likelihood estimates of undiagnosed HIV infections with different median incubation periods: 7.5, 10.0, and 12.3 years. C. Proportion of diagnosed infections out of the cumulative number of HIV infections, inclusive of AIDS cases. D. Proportion of diagnosed infections out of the cumulative number of HIV infections, excluding AIDS cases. E. Maximum likelihood estimates of undiagnosed HIV infections by sex, with different median incubation periods: 7.5, 10.0, and 12.3 years. Note that common logarithmic scale is used on the vertical axis. D. Proportion of diagnosed infections out of the cumulative number of HIV infections, excluding AIDS cases, by sex. 

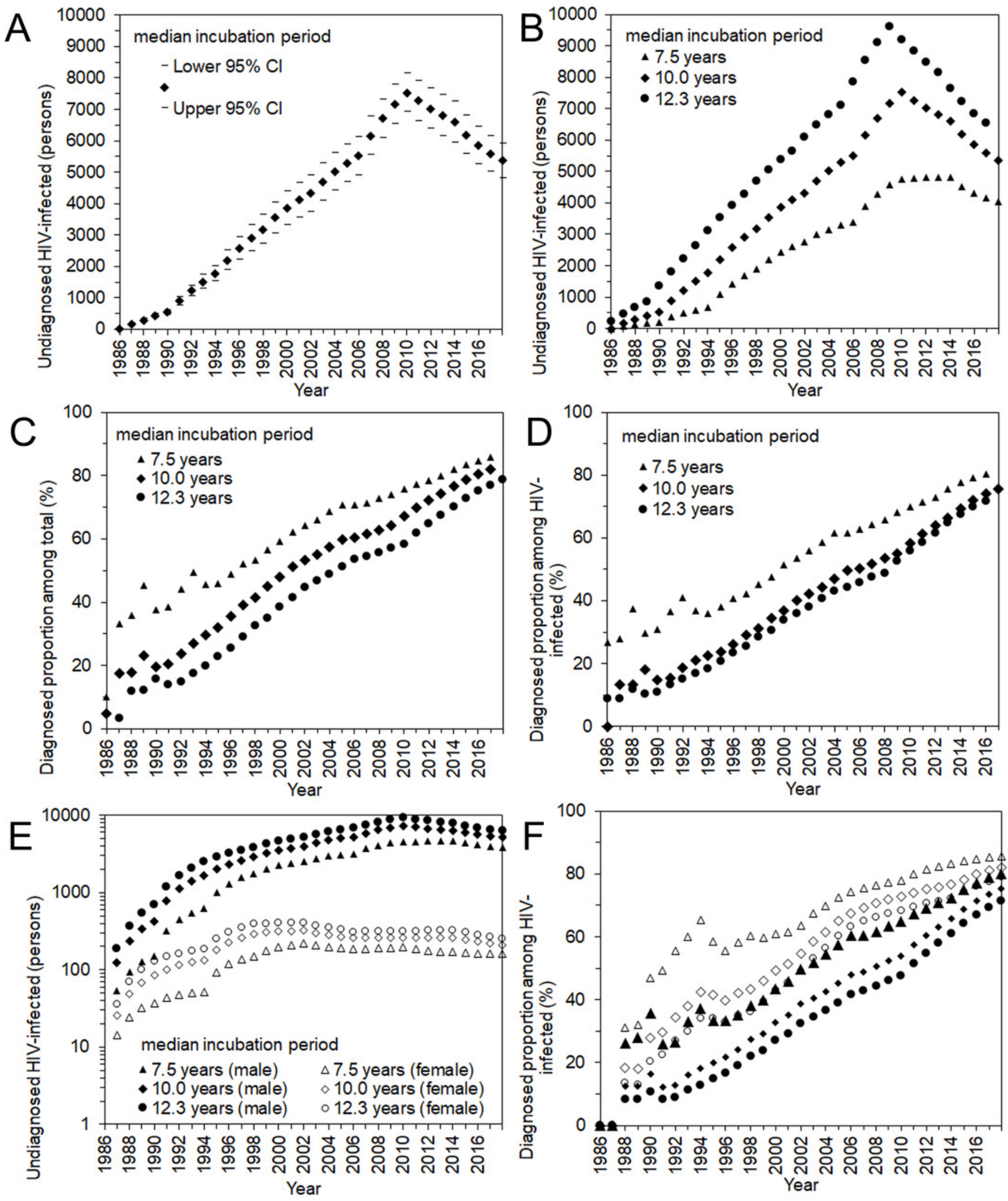


\section{Figure 5}

Estimated undiagnosed HIV infections and proportion of diagnosed infections at the end of 2017.

A. Estimates of undiagnosed HIV infections with different incubation periods. Whiskers extend to lower and upper $95 \%$ confidence intervals derived using a parametric bootstrapping method. B. Proportion of diagnosed infections out of the cumulative number of HIV infections, excluding AIDS cases (solid circles) or including AIDS cases but subtracting 2,321 deaths (empty circles). Whiskers extend to lower and upper 95\% confidence intervals derived using a parametric bootstrapping method.
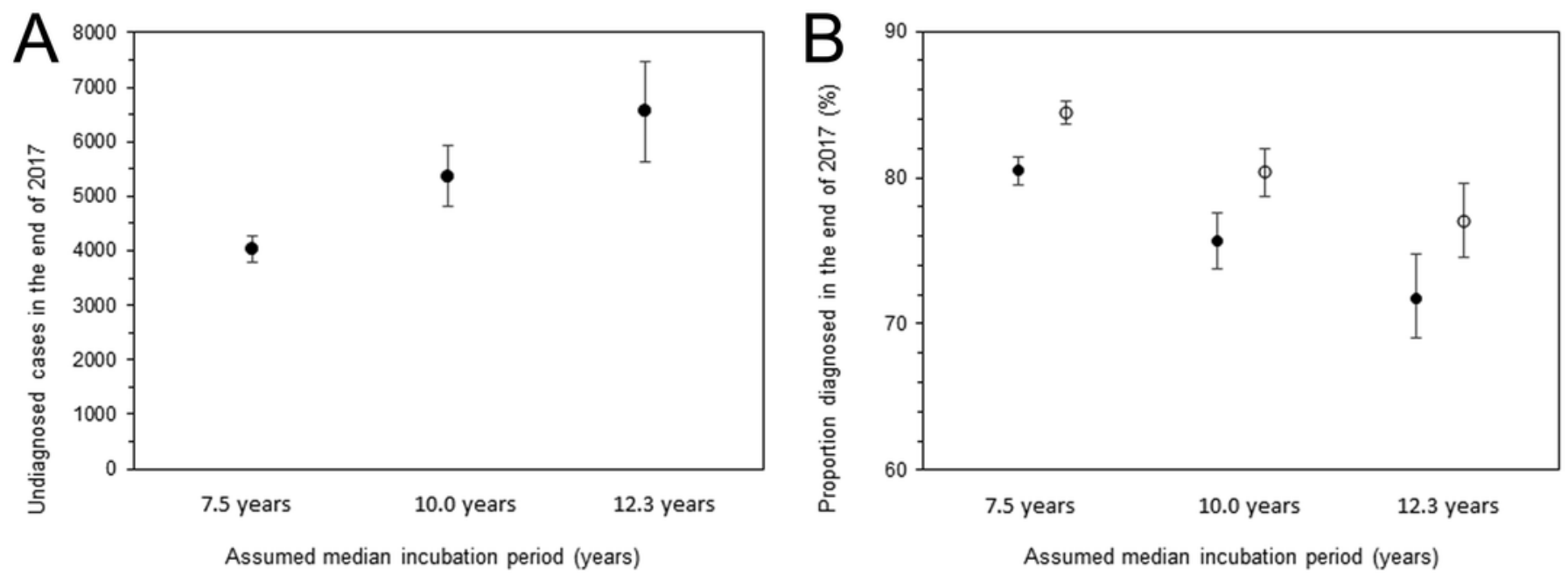